\title{
Content based Image Retrieval using Implicit and Explicit Feedback with Interactive Genetic Algorithm
}

\author{
Ghanshyam \\ Raghuwanshi \\ School of Information \\ Technology, RGPV, Bhopal
}

\author{
Nishchol Mishra \\ School of Information \\ Technology, RGPV, Bhopal
}

\author{
Sanjeev Sharma \\ School of Information \\ Technology, RGPV, Bhopal
}

\begin{abstract}
There are various new applications of genetic algorithms to information retrieval, mostly with respect to relevance feedback. However, they are yet to be evaluated in a way that allows them to be compared with each other and with other relevance feedback techniques. There is always need to efficiently store and retrieve image data to perform assigned tasks and to make a decision. This paper presents a new image retrieval framework with two types of relevance feedback i.e., implicit feedback in combination with explicit feedback. This paper employs Interactive Genetic Approach to discover a combination of descriptors that better characterizes the user perception of image resemblance. This approach provides better management and retrieval of images than the keyword-based approach. However, most of the conventional methods do not have the capability to effectively incorporate human interaction and emotion into retrieving images. In order to solve this problem we have developed an image retrieval system based on human preference and emotion by using an interactive genetic algorithm (IGA). In this approach we used two tier architecture of implicit and explicit feedback with IGA. Therefore, this system facilitates the search for the image not only with explicit queries, but also with implicit queries.
\end{abstract}

\section{Keywords}

Relevance feedback (RF), Geneticalgorithm (GA), Image Retrieval (IR).

\section{INTRODUCTION}

An image retrieval [1] system is a system for browsing, searching and retrieving images from a large database. User first enters his input to search in the database and then similarity matching function is performed between user input and large database. For this, the text- and content based approaches are used; they are usually adopted in image retrieval. In the text-based system, the images are manually annotated by text descriptors and then used by a database management system to perform image retrieval. And content based retrieval has been frequently used by the users. The primary goal of the CBIR system is to construct meaningful descriptions of physical attributes from images to facilitate efficient and effective retrieval [1], [2].In content based scheme, a user provides example images and then similar images are returned by indexing visual representation of images in a large scale database. But these methods of image retrieval do not sufficient for retrieving good results. Manual image annotation is time-consuming, laborious and expensive; to solve this, there has been a lot of research work done on automatic image annotation. Image retrieval can be defined as searching in a large image database space to bring relevant documents or images to users. It is understood, however, that this is not an easy task to do.
Because of the complexity of document writing styles and the difficulty users have in presenting their information requests, the retrieval results often frustrate users because they do not contain exact information what is required by the user. System with a modified query becomes unavoidable if a user wants to improve the retrieval results. Most systems provide little or no guidance to the user for modifying the original query. For solving this problem, relevance feedback is used to help users. The thought behind relevance feedback is to take the initial results that are returned from a given query and to use information about whether or not those results are relevant to perform a new query. Initially most work paying attention on improving the quality of search results from the relevance aspect, i.e., boosting the ranks of relevant images. This kind of methods is called relevance based re-ranking [3] [4] [5] [6]. There are generally three most popular feedback techniques.

\section{- Explicit Feedback:}

Explicit feedback is obtained from users indicating the relevance of a document retrieved for a query. This feedback is applied only when user is not satisfied with the retrieved result. This kind of feedback is called as explicit only when the other evaluators of a system know that the feedback provided is interpreted as relevance judgments.

\section{- Implicit Feedback:}

Implicit feedback is incidental from user actions, such as observing which documents they do and do not select for viewing, also the period of time spent in viewing a document, or page browsing or scrolling events. Generally implicit information is stored in the log files. That is log files are the place where history of the user interaction with the system is stored and this information acts as a key element in the feedback process. The basic differences of implicit feedback from that of explicit feedback are following:

1. The user is not assessing relevance for the advantage of the Information Retrieval system, but only satisfying their own needs. 2. There is no direct interaction of user in implicit feedback process because information is collected from log files while in explicit feedback user has to take participate in feedback process directly.

\section{- Blind Feedback:}

Blind feedback is also known as Pseudo relevance feedback. Relevance feedback provides a method for automatic local analysis, because of that user gets better retrieval result without an extended interaction.Although relevance feedback improves the performance of the retrieval system but its applicability 
still having some drawbacks [7].However the excess use of feedback technique with the retrieval method needs more processing time. However, feedback techniques also required to be improved. That's why a new technique is needed which is called Genetic algorithm which must be combined with the image retrieval system to improve the efficiency and also to optimize the search time. Here IGA is employed with the combination of implicit and explicit feedback in this approach.

\section{$>$ Genetic Algorithm}

A Genetic Algorithm (GA) is a method used to getquite accurate solutions to search problems through application of the values of evolutionary biology. Genetic algorithms (GAs) are not new to image retrieval systems [8][9]. A GA is an Artificial Intelligence technique which uses interactive methods to solve problems of searches that satisfies certain requirements. GAs have been broadlyvaluable in many areas of engineering such as signal processing, system identification, image retrieval and data mining problems [10][11][12][13]. In [14], GAs is applied to exercise difficulty-level adaptation in schools and universities with very good results. Beligianniset al.[15] applied GAs to the problem of intelligent medical diagnosis of male incompetence. Wu et al.[16] proposed a genetic-based solution for a coordinate transformation test of Global Positioning System positioning. Pan [17] designed robust D-stable IIR filters by using GAs with embedded stability criterion. The GA consists of an iterative process that evolves a working set of individuals called a populationtoward an objective function, or fitness function.GAs also have been successfully applied in the research of CBIR [18][19][20]. Working of GA is shown in figure (1). There are the three terms used in Genetic algorithm as follows:

- Crossover - exchange of genetic material (substrings) denoting rules, structural components, features of a machine learning, search, or optimization problem. Each individual must then be evaluated to produce a fitness function. Fitness functions refer to a quantifiable scalar-valued measure of the fitness of an individual. In the case of business processes the fitness functions of each process will most likely be generated by evaluating the time it would take to complete each process. Faster processes would receive high fitness functions, and slower ones would receive lower functions.

- Selection - the application of the fitness criterion to choose which individuals from a population will go on to reproduce. Once each individual has been evaluated, the individuals with the highest fitness functions will be combined to produce a second generation. In general the second generation of individuals can be expected to be 'fitter' than the first, as it was derived only from individuals carrying high fitness functions.

- $\quad$ Replication - the propagation of individuals from one generation to the next.

\footnotetext{
Begin

$\mathrm{i}=0$;

Initialize S(i);

Evaluate S(i);

While (not termination condition)

Do

Begin
}

$\mathrm{i}=\mathrm{i}+1$;

Select s(i) from S(i-1);

After (cross and mutation)

S(i);

Evaluate S (i);

end;

end;

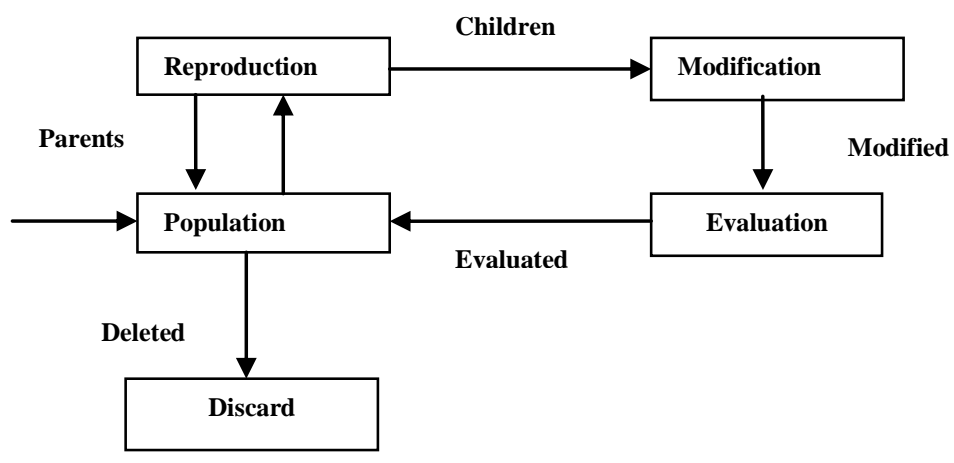

Figure 1: block diagram of the working of Genetic Algorithm

Interactive Genetic Algorithm:

Interactive Genetic Algorithm (IGA) is a branch of evolutionary computation. The basic difference between IGA and GA is the creation of the fitness function, that is, the fitness is determined by the user's valuation and not by the predefined mathematical function.A user can interactively determine which members of the population will produce again, and IGA automatically generates the next generation of content based on the user's input. During repeated rounds of content creation and fitness assignment, IGA enables unique content to grow that suits the user's choices. Based on this reason, IGA can be used to solve problems that are difficult or impossible to devise a computational fitness function, for example, evolving images, music, various artistic designs, and forms to fit a user's visual choices. From figure this is clear that firstly user puts a query image and then matching function is used which matches the query image with the database image. Images are compared and then user evaluates the retrieved results if he is not satisfied then this leads to the other evaluations and also iterative Genetic algorithm. This process continuous until the user is not satisfied. An Information Retrieval System is basically constituted by three main components, the documentary database, and query subsystemThe matching mechanismwhose composition is introduced in [21][22].

\section{RELATED WORK}

The authors in [23] considered the RGB color space and adopted the color distributions, as well as the image bitmap, as the visual features for image retrieval. This approach was quite efficient and opened the door for other researchers in this field of image retrieval. In [24], the YUV color space is used, and discrete wavelet transform is applied to extract four types of features i.e., approximations, horizontal details, vertical details, and diagonal details at each wavelet level. It produces some different results than previous one on the same database. This approach was not too good because its experimental result shows low precision values than the previous one. In [25]Author proposed a contentbased image retrieval method based on an interactive genetic algorithm (IGA). The mean value and the standard deviation of a color image are used as color features. In addition, author also considered the entropy based on the gray level co-occurrence matrix as the texture feature. Further, to bridge the gap between 
the retrieving results and the users' expectation, the IGA is employed such that the users can adjust the weight for each image according to their expectations. In this paper author used two types of feature.

\section{i) The Color Feature}

Each image in the database can be represented using three primaries of a color space. The most common color space is RGB. Thus, each pixel of a color image is represented by a vectorColor Image Retrieval Based on Interactive Genetic Algorithm.

$\mathrm{P}_{\mathrm{i}}=\left[\begin{array}{l}R i \\ G i \\ B i\end{array}\right]$

where $P i$ is the $i$ th pixel of the image, $1 \leq i \leq M$. Ri, Gi, and $B i$ are thecomponents of primary colors red, green, blue, respectively. The $M$ is the sizeof the image, and the components of Pi depict the color information.

The mean value $(\mu)$ and the standard deviation $(\sigma)$ of the color image aredetermined as follows:

$$
\begin{gathered}
\mu=\frac{1}{M} \sum_{i=1}^{M} P i \ldots \ldots \ldots(2) \\
\sigma=\left[\frac{1}{M-1} \sum_{i=1}^{M}(P i-\mu) 2\right]^{1 / 2} .(3)
\end{gathered}
$$

where $\mu=[\mu R \mu G \mu B] T$ and $\sigma=[\sigma R \sigma G \sigma B] T$, each component of $\mu$ and $\sigma$ indicatesthe RGB information, respectively.

\section{ii) The Texture Feature}

Texture is an important image feature that has been used for characterization ofimages. If choose appropriate texture descriptor, the performance of theCBIR must be improved. In this paper, the entropy is used to capture textureinformation in an image and is defined as follows.

$$
\operatorname{Entropy}(E)=-\sum_{i} \sum_{\mathrm{j}} \mathrm{C}(\mathrm{i}, \mathrm{j}) \log \mathrm{C}(\mathrm{i}, \mathrm{j}) \ldots(4)
$$

Where $C(i, j)$ is the gray level co-occurrence matrix. The $C(i, j)$ is obtainedby first specifying a displacement vector and then counting all pairs of pixelsseparated by the displacement and having gray levels $i$ and $j$.

In other paper author proposed, a user-oriented mechanism for CBIR method based on an interactive genetic algorithm (IGA). Color attributes like the mean value, the standard deviation, and the image bitmap of a color image are used as the features for retrieval. In addition, the entropy based on the gray level cooccurrence matrix and the edge histogram of an image is also considered as the texture features. Furthermore, to reduce the gap between the retrieval results and the users' expectation, the IGA is employed to help the users identify the images that are most satisfied to the users' need. In this paper the author used three types of descriptor. i).Color descriptor, ii).Texture descriptor ,iii).Edge descriptor.In another paper [26] author developed a wide variety of algorithms have been proposed to tackle reranking by emphasizing its different aspects. A study of the development of state-of-the-art reranking methods is able to facilitate our understanding of the essentials of visual reranking, offer a clear view of what user have achieved, and informhow to resolve emerging obstacles in future. As such, this paper presents an introduction of multimedia visual reranking, including its objective, features utilization, reranking strategy, and user interaction. Author used For relevance measurement, many criterions have beenproposed, e.g., precision, recall, non- interpolated averageprecision (AP) and normalized discounted cumulated gain (NDCG). The most popular ones in relevance based rerankingare AP and NDCG. The AP averages the precision valuesobtained when each relevant sample occurs.

$\mathrm{AP} @ \mathrm{k}=\frac{1}{\mathrm{Zk}} \sum_{i=1}^{k}[p(i) \times \operatorname{rel}(i)]$

Where $p(i)$ is the precision at rank $i$ and $\operatorname{rel}(i)$ is the binaryfunction on the relevance of the $i$-th ranked sample with " 1 " forrelevant and " 0 " for irrelevant. This is a normalizationconstant that is chosen to guarantee $\mathrm{AP} @ k=1$ for a perfectranking result.

\section{PROPOSED APPROACH}

After studying many image retrieval algorithms, it is found that they all are suffering their own advantages and disadvantages. So we concluded a new approach which is based on relevance feedback and interactive genetic algorithm. Here we used only two types of relevance feedback:

1. Implicit feedback: it is useful because there is no need of direct human interaction in feedback process and required information can be collected from the log files i.e. history stored in the files. But it is also suffering the disadvantage of noisy data stored in the files. Because of that it is not advantageous in all the cases.

2. Explicit feedback: in this direct user interaction is available in the case if user is not satisfied by the result of search engine. User just has to put another hint or input any image or data and wait for the response of the search engine.

\section{Algorithm}

1. Start

2. Initially feature extraction of all the images of the database is performed.

3. User inputs a query image (Query by example).

4. The interactive genetic algorithm requires a population of potential solutions to be initialized at the beginning of the GA process. Usually, the initialization process varies with the applications; here, we take up the images generated by the first query as initial candidate images.

Feature extraction of query image i.e. color, texture, and edge are extracted by using of following formulas:

$$
\begin{gathered}
\qquad \mathrm{P}_{\mathrm{i}}=\left[\begin{array}{l}
R i \\
G i \\
B i
\end{array}\right] \\
\text { Mean value } \mu=\frac{1}{M} \sum_{i=1}^{M} P i \\
\text { Standard deviation } \sigma=\left[\frac{1}{M-1} \sum_{i=1}^{M}(P i-\mu) 2\right]^{1 / 2} \\
\operatorname{Entropy}(E)=-\sum_{i} \sum_{\mathrm{j}} \mathrm{C}(\mathrm{i}, \mathrm{j}) \log \mathrm{C}(\mathrm{i}, \mathrm{j})
\end{gathered}
$$

Where $\mu, \sigma$ are used for color and entropy is used for texture information. And Edge Histogram Descriptor (EHD) is used for extracting edge information.

5. Extracted features are compared with the stored feature of the images in the database i.e. similarity matching function is applied here.The fitness function is working to calculate the quality of the chromosomes in the population. The use of IGA allows the combination of human and computer efforts for problem solving [27]. In our approach, the quality of a chromosome $C$ with relation to the query $q$ is defined as

$F(q, C)=w 1 \cdot \operatorname{sim}(q, C)+w 2 \cdot \delta+$ implicitfeed,

wheresim $(q, C)$ represents the similarity measure between images, $\delta$ indicates the impact factor of 
human's decision, the coefficients $w 1$ and $w 2$ determine the relative importance of them to calculate the fitness. Initially value of $\delta$ is set to zero and value of implicitfeed is zero if the search for the same query image is not performed before else Information through the log files are matched at the same time and relevant images are extracted.

6. Result is displayed.

7. If user satisfied then searching get finished

8. Else if user does not satisfied then explicit feedback with interactive genetic algorithm works here and similarity function is again calculated by adjusting the value of impact factor of human judgment $\delta$.

9. User puts his feedback after examining the searched result

10. User repeats this step until and unless he is not satisfied.

11. End

We know that data stored in the log files are same at particular time and there may be slight change in the $\log$ data after some time. That's why there is no need of searching the log files again for a single search. This algorithm would work on the following conditions:

1. Implicit feedback would be executed only once.
2. Explicit Feedback would run zero time, one time or more, depending upon the request for image is only fulfilled directly by search engine, or by implicit feedback, or by one or more time explicit feedback.

Two main factors of interactive genetic algorithm are initial population and fitness. In Initial populationthis algorithm requires a population of possible solutions to be initialized at the beginning of the GA process. Working procedure of our approach is shown in figure (2).Usually, the initialization process varies with the applications; here, we accept the first query results of a sample image as initial candidate images. The fitness function is used to calculate the quality of the chromosomes in the population. The use of IGA allows the combination of human and computer efforts for problem solving. Because the goal of our proposed system is to retrieve the images that are most satisfied to the users' need.Here concept of InteractiveGenetic algorithm when merge with relevance feedback approach helps in improving the efficiency and decreasing the search time. Results are generated in the decreasing order of their relevancy. Most relevant images come first in the list then less relevant images come.Our approach is better than other IGA approaches In that we are also using implicit feedback in combination with explicit feedback. This combination produces better results then only explicit feedback. Implicit feedback is the beauty of our approach.

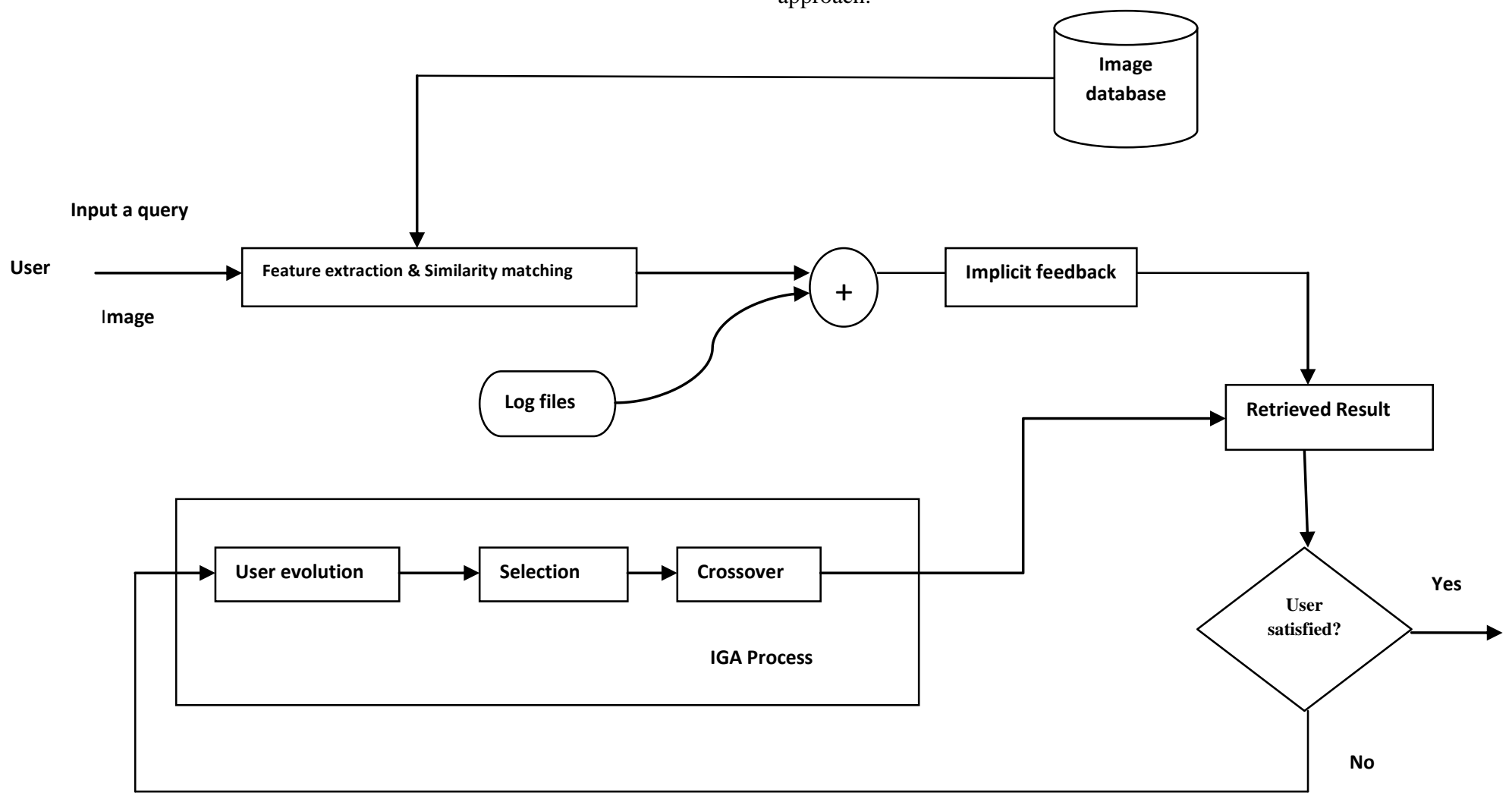

Figure 2: Block diagram of the two tier architecture of Image retrieval using InteractiveGenetic Algorithm

\section{RESULT}

Initially feature extraction of all the images, stored in the database is performed and all the extracted features are stored separately. Now query image is loaded by just browsing the image from anywhere in the system as shown in figure (3). After this feature extraction of the query image takes place. 


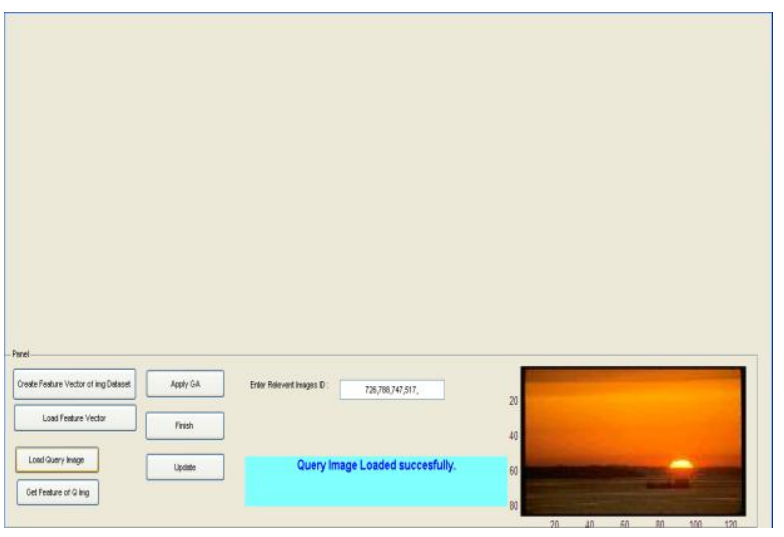

Figure (3): initially query image is loaded

Now the similarity measurement is done in tow steps:In first step similarity measurement of the features of query image with the stored features of the database images and in second step with the stored information about the retrieval history of the images in the log files Retrieving of information from logfiles is also called implicit feedback. Then retrieved related images are shown in figure (4). Images are retrieved in the decreasing order of their relevancy, this means higher relevant images are at the top and less relevant images are at the bottom.

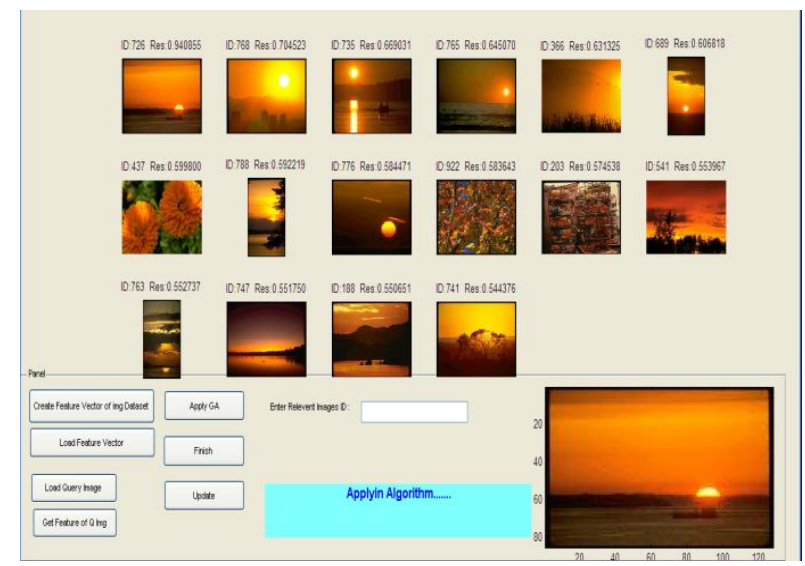

Figure(4): After feature extraction and similarity matching, result is displayed

After this step there may be two situations either user gets satisfied with the retrieved result or user does not get satisfied with the retrieved result,if user is not satisfied then he can apply his own feedback by improving the query. This is also called explicit feedback. Now user simply put the ids of the retrieved images which he considers more relevant and now searching is again done on the basis of improved query as shown in figure(5). If user again does not satisfied with the retrieved result then he can again apply his feedback as shown in figure (d). This process repeats until user gets satisfied.

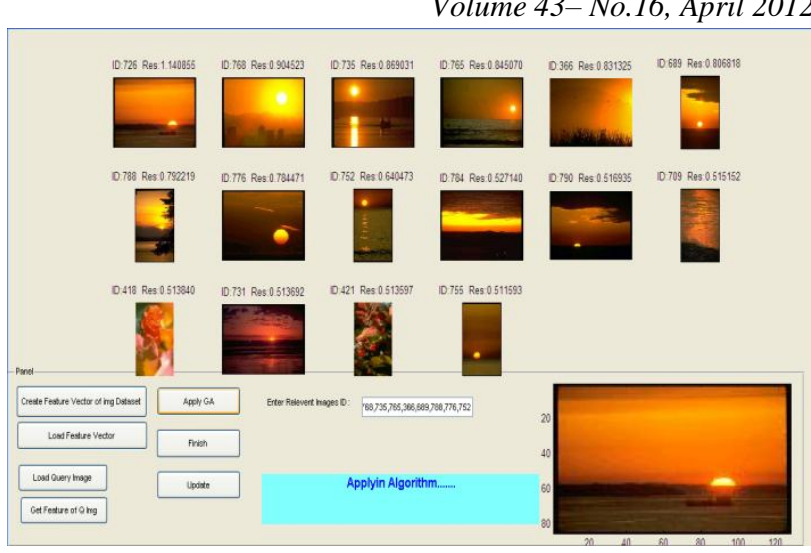

Figure(5): Explicit feedback is applied and result is displayed

Precision and recall

We can evaluate the performance of our algorithm by examining the retrieved relevant images to the query image. The effectiveness of the retrieval can be defined in terms of the precision and recall rate. Precision and recall can be defined as follows

$$
\begin{aligned}
& \text { Precision }=\frac{\mathrm{N}_{A}(\mathrm{q})}{\mathrm{N}_{R}(\mathrm{q})} \\
& \text { Recall }=\frac{\mathrm{N}_{A}(\mathrm{q})}{\mathrm{N}_{t}}
\end{aligned}
$$

Where $\mathrm{N}_{A}(\mathrm{q})$ denotes the number of relevant images similar to the query image, $\mathrm{N}_{R}(\mathrm{q})$ shows the total number of relevant images in the response to the query and $\mathrm{N}_{t}$ denotes the total number of images available in the database. Here we took top 16 images to compute the precision and recall. We carried out the experiment for 20 times on some images of database. After this, calculation of precision and recall is performed. Here one point can be noted that, after some image search log file stores the information of human interaction with the system i.e., when user makes same search which has been made by him or someone else previously, then best result is retrieved through the log files. This is the beauty of our approach. Experimental results are shown in the table. In table(a) and table (b) results are shown.

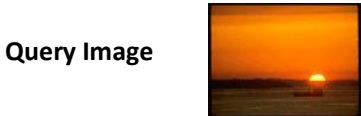

\begin{tabular}{|c|c|}
\hline Image_ID & $\begin{array}{c}\text { Similarity } \\
\text { measure before } \\
\text { explicit feedback }\end{array}$ \\
\hline 726 & 0.940855 \\
\hline 768 & 0.704523 \\
\hline 735 & 0.669031 \\
\hline 765 & 0.64507 \\
\hline 366 & 0.631325 \\
\hline 689 & 0.606818 \\
\hline 437 & 0.5998 \\
\hline 788 & 0.592219 \\
\hline 776 & 0.584471 \\
\hline 922 & 0.583643 \\
\hline
\end{tabular}

Table (a) : Similarity measure before explicit feedback

\begin{tabular}{|c|c|}
\hline Image_ID & $\begin{array}{c}\text { Similarity } \\
\text { measure after } \\
\text { explicit feedback }\end{array}$ \\
\hline 726 & 1.040855 \\
\hline 768 & 0.804523 \\
\hline 735 & 0.769031 \\
\hline 765 & 0.74507 \\
\hline 366 & 0.731325 \\
\hline 689 & 0.706818 \\
\hline 788 & 0.692219 \\
\hline 776 & 0.684471 \\
\hline 517 & 0.544222 \\
\hline 186 & 0.543967 \\
\hline
\end{tabular}

Table (b) : Similarity measure after explicit feedback 


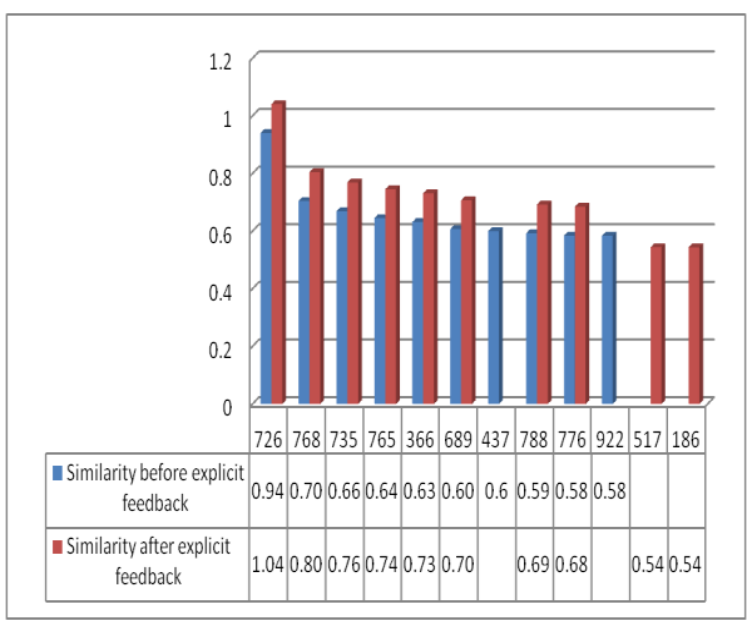

Figure 6: Graph corresponding to table(a) \& table(b).

\section{CONCLUSION AND FUTURE WORK}

In this paper we have presented a new approach which is based on user oriented support with interactive genetic algorithm. Here we have created two tier architecture of implicit and explicit feedback. Conventional methods are based on visual features which are not producing efficient result but our approach reduces the gap between the visual features and human perception. Combination of implicit and explicit feedback in two tier architecture produces better result than only explicit feedback. The color attribute, mean value and standard deviation are used. Entropy and edge orientation histograms are used as a texture descriptor. Experimental results of proposed system prove the effectiveness of the image retrieval and major improvement in the retrieval performance. So in brief, our approach shows good tuning of IGA with the implicit and explicit feedback, thus the result generated is also satisfactory at low cost. Our future work will emphases on filtering out the noisy data from $\log$ files at regular intervals of time and also maintains the history of user interaction with the retrieval system in an efficient way.

\section{REFERENCES}

[1] M. Antonelli, S. G. Dellepiane, and M. Goccia, "Design and implementation of Web-based systems for image segmentation and CBIR," IEEETrans. Instrum. Meas., vol. 55, no. 6, pp. 1869-1877, Dec. 2006.

[2] N. Jhanwar, S. Chaudhuri, G. Seetharaman, and B. Zavidovique, "Content based image retrieval using motif co occurrence matrix," Image Vis.Comput., vol. 22, no. 14, pp. 1211-1220, Dec. 2004.

[3] W. H. Hsu, L. S. Kennedy and S.-F. Chang. Reranking Methods for Visual Search. IEEE Multimedia, vol. 14, no. 3, pp. 14-22, 2007.

[4] Y. Jing and S. Baluja. Visualrank: Applying pagerank to large-scale image search. IEEE Trans. Pattern Analysis and Machine Intelligence, vol. 30, pp. 1877-1890, 2008.

[5] T. Yao, T. Mei and C.-W. Ngo. Co-reranking by mutual reinforcement for image search. CIVR, pp. 34-41, 2010.

[6] X. Tian, L. Yang, J. Wang, Y. Yang, X. Wu and X.-S. Hua. Bayesian Video Search Reranking. ACM Int'l Conf. Multimedia, pp. 131-140, 2008.
[7] ] J. Han, K. N. Ngan, M. Li, and H.-J. Zhang, "A memory learning framework for effective image retrieval," IEEE Trans. Image Process., vol. 14, no. 4, pp. 511-524, Apr. 2005.

[8] ] H. Chen, "Machine learning for information retrieval: neural networks,symbolic learning, and genetic algorithms". Journal of the American Society for Information Science, 46(3), 1995, pp. 194-216.

[9] J. Savoy and D. Vrajitoru, "Evaluation of learning schemes used in information retrieval (CR-I-95-02)". Universite de Neuchatel, Facultededroitet des Sciences Economiques, 1996.

[10] G. Beligiannis, L. Skarlas, and S. Likothanassis, "A generic applied evolutionaryhybrid technique for adaptive system modeling and information mining," IEEE Signal Process. Mag._Special Issue on "Signal Processing for Mining Information”, vol. 21, no. 3, pp. 28-38, May 2004.

[11] G. N. Beligiannis, L. V. Skarlas, S. D. Likothanassis, and K. G. Perdikouri, "Nonlinear model structure identification of complex biomedical data using a genetic-programmingbased technique," IEEE Trans. Instrum. Meas., vol. 54, no. 6, pp. 2184-2190, Nov. 2005.

[12] C.-Y. Chang and D.-R. Chen, "Active noise cancellation without secondarypath identification by using an adaptive genetic algorithm," IEEE Trans. Instrum. Meas., vol. 59, no. 9, pp. 2315-2327, Sep. 2010.

[13] S. Osowski, R. Siroic, T. Markiewicz, and K. Siwek, "Application of support vector machine and genetic algorithm for improved blood cell recognition," IEEE Trans. Instrum. Meas., vol. 58, no. 7, pp. 2159-2168, Jul. 2009.

[14] C. Koutsojannis, G. Beligiannis, I. Hatzilygeroudis, C. Papavlasopoulos, and J. Prentzas, "Using a hybrid AI approach for exercise difficulty level adaptation," Int. J. Continuing Eng. Educ. Life-Long Learn., vol. 17, no. 4/5, pp. 256-272, 2007.

[15] G. Beligiannis, I. Hatzilygeroudis, C. Koutsojannis, and J. Prentzas, "A GA driven intelligent system for medical diagnosis," in Proc. KES, vol. 4251. Heidelberg, Germany: Springer-Verlag, 2006, pp. 968-975.

[16] C.-H. Wu, H.-J. Chou, and W.-H. Su, "A genetic approach for coordinate transformation test of GPS positioning," IEEE Geosci. Remote Sens. Lett., vol. 4, no. 2, pp. 297 301, Apr. 2007.

[17] S.-T. Pan, "Design of robust D-stable IIR filters using genetic algorithms with embedded stability criterion," IEEE Trans. Signal Process., vol. 57, no. 8, pp. 3008-3016, Aug. 2009.

[18] G. Paravati, A. Sanna, B. Pralio, and F. Lamberti, “A genetic algorithm for target tracking in FLIR video sequences using intensity variation function," IEEE Trans. Instrum. Meas., vol. 58, no. 10, pp. 3457-3467, Oct. 2009.

[19] S. F. da Silva, M. A. Batista, and C. A. Z. Barcelos, "Adaptive image retrieval through the use of a genetic algorithm," in Proc. 19th IEEE Int.Conf. Tools WithArtif. Intell., 2007, pp. 557-564.

[20] Z. Steji, Y. Takama, and K. Hirota, "Genetic algorithmbased relevance feedback for image retrieval using local similarity patterns," Inf. Process.Manage., vol. 39, no. 1, pp. 1-23, Jan. 2003. 
[21] R. Baeza-Yates and B. Ribeiro-Neto. Modern Information Retrieval, Adisson, 1999.

[22] G. Salton and M.H. McGill. Introduction to Modern Information Retrieval, McGraw-Hill, 1983.

[23] T.-C. Lu and C.-C. Chang, "Color image retrieval technique based on color features and image bitmap," Inf. Process. Manage., vol. 43, no. 2, pp. 461-472, Mar. 2007.

[24] T.-W. Chiang and T.-W. Tsai, "Content-based image retrieval via the multiresolution wavelet features of interest," J. Inf. Technol. Appl., vol. 1, no. 3, pp. 205-214, Dec. 2006.
[25] Chih-Chin Lai, "A User-Oriented Image Retrieval System Based on Interactive Genetic Algorithm,"IEEE TRANSACTIONS ON INSTRUMENTATION AND MEASUREMENT/0018-9456/2011.

[26] [26]XinmeiTian, "Multimedia Visual Reranking - from Ranking Objectives to Learning Strategies" Manuscript received October, 2010, revised February, 2011, accepted April, 2011.

[27] H. Takagi, "Interactive evolutionary computation: Fusion of the capacities of EC optimization and human evaluation," Proc. IEEE, vol. 89, no. 9, pp. 1275-1296, Sep. 2001. 